\title{
STRONG TIME-PERIODIC SOLUTIONS TO THE BIDOMAIN EQUATIONS WITH ARBITRARY LARGE FORCES
}

\author{
YOSHIKAZU GIGA, NAOTO KAJIWARA, AND KLAUS KRESS
}

\begin{abstract}
We prove the existence of strong time-periodic solutions to the bidomain equations with arbitrary large forces. We construct weak time-periodic solutions by a Galerkin method combined with Brouwer's fixed point theorem and a priori estimate independent of approximation. We then show their regularity so that our solution is a strong time-periodic solution in $L^{2}$ spaces. Our strategy is based on the weak-strong uniqueness method.
\end{abstract}

\section{INTRODUCTION}

In this paper, we consider the bidomain system which is a well-established system describing the electrical wave propagation in the heart. The system is given by

$(\mathrm{BDE})$

$$
\left\{\begin{array}{cl}
\partial_{t} u-\operatorname{div}\left(\sigma_{i} \nabla u_{i}\right)+f(u, w)=s_{i} & \text { in }(0, \infty) \times \Omega, \\
\partial_{t} u+\operatorname{div}\left(\sigma_{e} \nabla u_{e}\right)+f(u, w)=-s_{e} & \text { in }(0, \infty) \times \Omega, \\
\partial_{t} w+g(u, w)=0 & \text { in }(0, \infty) \times \Omega, \\
u=u_{i}-u_{e} & \text { in }(0, \infty) \times \Omega, \\
\sigma_{i} \nabla u_{i} \cdot \nu=0, \sigma_{e} \nabla u_{e} \cdot \nu=0 & \text { on }(0, \infty) \times \partial \Omega, \\
u(0)=u_{0}, w(0)=w_{0} & \text { in } \Omega .
\end{array}\right.
$$

Here $\Omega \subset \mathbb{R}^{d}$ denotes a domain describing the myocardium and the outward unit normal vector to $\partial \Omega$ is denoted by $\nu$. The unknown functions $u_{i}$ and $u_{e}$ model the intra- and extracellular electric potentials, and $u$ denotes the transmembrane potential. The variable $w$, the so-called gating variable, corresponds to the ionic transport through the cell membrane. The anisotropic properties of the intra- and extracellular tissue parts are described by the conductivity matrices $\sigma_{i}(x)$ and $\sigma_{e}(x)$, whereas $s_{i}(t, x)$ and $s_{e}(t, x)$ denote the intra- and extracellular stimulation current, respectively. The ionic transport is described by the nonlinearities $f$ and $g$. In this article, we will consider a large class of ionic models including those by FitzHugh-Nagumo, Rogers-McCulloch, and Aliev-Panfilov. Note, that we will look at the Aliev-Panfilov model in a slightly modified form as considered, e.g., in [13]. The FitzHugh-Nagumo model reads as

$$
\begin{aligned}
& f(u, w)=u(u-a)(u-1)+w=u^{3}-(a+1) u^{2}+a u+w, \\
& g(u, w)=-\varepsilon(k u-w),
\end{aligned}
$$

with $0<a<1$ and $k, \varepsilon>0$.

In the Rogers $-M c$ Culloch model the functions $f$ and $g$ are given by

$$
\begin{aligned}
& f(u, w)=b u(u-a)(u-1)+u w=b u^{3}-b(a+1) u^{2}+b a u+u w, \\
& g(u, w)=-\varepsilon(k u-w),
\end{aligned}
$$

Key words and phrases. bidomain model, strong periodic solutions, weak-strong uniqueness, large data.

This work was partly supported by the DFG International Training Group IRTG 1529 and the JSPS Japanese-German Graduate Externship on Mathematical Fluid Dynamics. The first author is partly supported by JSPS through grant Kiban S (No. 26220702), Kiban A (No.17H01091), Kiban B (No.16H03948). The second author is a research assistant supported by Kiban S (No. 26220702). The third author is supported by the DFG International Research Training Group 1529 on Mathematical Fluid Dynamics at TU Darmstadt . 
with $0<a<1$ and $b, k, \varepsilon>0$.

For the modified Aliev-Panfilov model we have

$$
\begin{aligned}
& f(u, w)=b u(u-a)(u-1)+u w=b u^{3}-b(a+1) u^{2}+b a u+u w, \\
& g(u, w)=\varepsilon(k u(u-1-d)+w)
\end{aligned}
$$

with $0<a, d<1$ and $b, k, \varepsilon>0$. To get weak time-periodic solutions for the Aliev-Panfilov model we will need the further assumption $b>k$. For a detailed description of the bidomain model we refer to the monographs by Keener and Sneyd [17] and Colli Franzone, Pavarino, and Scacchi [5].

Since the bidomain model describes electrical activities in the heart, it is a natural question to ask whether it admits time-periodic solutions. Therefore, consider the situation where the bidomain model is innervated by periodic intra- and extracellular stimulation currents $s_{i}$ and $s_{e}$. Recently, Hieber, Kajiwara, Kress, and Tolksdorf [9] proved the existence and uniqueness of a strong $T$-periodic solution to the innervated model in real interpolation spaces provided the external forces $s_{i}$ and $s_{e}$ are both time-periodic of period $T>0$. In their approach, they furthermore assumed that the external forces satisfy a suitable smallness condition.

It is the goal of this paper to prove the existence of time-periodic solutions without assuming any smallness condition on the external forces. We employ the method given by Galdi, Hieber, and Kashiwabara [12] for the case of the primitive equations. First, the existence of weak time-periodic solutions is shown by using a Galerkin approximation combined with Brouwer's fixed point theorem. Then, we use the global well-posedness result by Colli Franzone and Savaré [7] and consider the weak time-periodic solution as a weak solution to the initial value problem. Finally, we apply a weak-strong uniqueness argument to get a strong-time periodic solution without assuming any smallness condition for the external applied currents.

The bidomain model was first introduced by Tung [21] in 1978. Despite its central importance in cardiac electrophysiology, the rigorous mathematical analysis started not until the work of Colli Franzone and Savaré [7] in 2002. They introduced a variational formulation of the bidomain problem and proved the existence and uniqueness of weak and strong solutions to the bidomain equations with FitzHugh-Nagumo type nonlinearities. A slightly more detailed review of their results is given in Section [4. Veneroni [22] extended their results to more general ionic models including the Luo and Rudy I model. In 2009, Bourgault, Cordière, and Pierre [3] presented a new approach to the bidomain system. They introduced the so-called bidomain operator within the $L^{2}$-setting and showed that it is a non-negative and selfadjoint operator. By using the bidomain operator, they transformed the bidomain system into an abstract evolution equation and showed the existence and uniqueness of a local strong solution and the existence of a global weak solution for a large class of ionic models including the three models introduced above. Later, Kunisch and Wagner [18] showed uniqueness and further regularity for these weak solutions. Giga and Kajiwara [11] investigated the bidomain system within the $L^{p}$ setting and showed that the bidomain operator is the generator of an analytic semigroup on $L^{p}(\Omega)$ for $p \in(1, \infty]$. Recently, Hieber and Prüss proved the maximal $L_{p}-L_{q}$ regularity for the bidomain operator in [15] and proved the global well-posedness in [14]. This paper considered the case $s_{i, e}=0$ and only FitzHugh-Nagumo type non-linearities. The general case was treated in [16]. However, to use the global well-posedness results in [16], we need higher regularity for $s_{i, e}$ compared with [7]. More recently, the bidomain equations were treated as a kind of gradient system in [2]. They proved the global well-posedness results in $L^{2}$ spaces and energy spaces. Their paper also treated the case $s_{i, e}=0$.

For results concerning the dynamics of the solution, we refer to the work of Mori and Matano [19]. They studied the stability of front solutions of the bidomain equations.

On a microscopic level, the cardiac cellular structure is described by two disjoint domains $\Omega_{i}$ and $\Omega_{e}$, which denote the intra- and extracellular space, respectively, and which are separated by the the active membrane $\bar{\Gamma}=\partial \Omega_{i} \cap \partial \Omega_{e}$. The intra- and extracellular quantities are defined on the corresponding domains and the transmembrane potential $u$ is a function on $\bar{\Gamma}$. After a homogenization procedure, see, e.g., $[6,7]$, the macroscopic model of the bidomain equations is obtained. Here all membrane, intra-, and extracellular quantities are defined everywhere on $\Omega$. 
This paper is organized as follows: We start in Section 2 with collecting known facts concerning the bidomain operator. In Section 3, we construct a weak time-periodic solution to the bidomain equations. When we construct the weak time-periodic solutions, we need some growth conditions on the nonlinear terms $f, g$. Fortunately, all three models mentioned above fulfill these conditions, which are confirmed in the appendix. A global well-posedness result is reviewed in Section 4 and invoked to obtain a strong time-periodic solution for the FitzHugh-Nagumo model. We do not treat the other two models introduced above since the global well-posedness for the initial value problem is not proved in a suitable $L^{2}$ setting.

\section{Preliminaries}

In this section, we fix some notation and formally introduce the bidomain operator in a weak as well as in a strong setting. In the whole article, let $\Omega \subset \mathbb{R}^{d}$ denote a bounded domain whose boundary $\partial \Omega$ is of class $C^{2}$. For convenience, we use the following notation for the function spaces which we will use throughout this article

$$
V=H^{1}(\Omega), H=L^{2}(\Omega), V^{\prime}=\left(H^{1}(\Omega)\right)^{\prime},
$$

where the spaces are endowed with their usual norms and they are Hilbert spaces. Furthermore, we set $Q=(0, T) \times \Omega$. The canonical pair of $V^{\prime}$ and $V$ is denoted by $V^{\prime}\langle\cdot, \cdot\rangle_{V}$.

We assume that the conductivity matrices $\sigma_{i}$ and $\sigma_{e}$ satisfy the following assumption.

Assumption C. The conductivity matrices $\sigma_{i}, \sigma_{e}: \bar{\Omega} \rightarrow \mathbb{R}^{d \times d}$ are symmetric matrices and are functions of class $C^{1}(\bar{\Omega})$. Ellipticity is imposed by means of the following condition: there exist constants $\underline{\sigma}, \bar{\sigma}$ with $0<\underline{\sigma}<\bar{\sigma}$ such that

$$
\underline{\sigma}|\xi|^{2} \leq{ }^{t} \xi \sigma_{i}(x) \xi \leq \bar{\sigma}|\xi|^{2} \text { and } \quad \underline{\sigma}|\xi|^{2} \leq{ }^{t} \xi \sigma_{e}(x) \xi \leq \bar{\sigma}|\xi|^{2}
$$

for all $x \in \bar{\Omega}$ and all $\xi \in \mathbb{R}^{d}$. Moreover, it is assumed that

$$
\begin{aligned}
& \sigma_{i} \nabla u_{i} \cdot \nu=0 \quad \Leftrightarrow \quad \nabla u_{i} \cdot \nu=0 \quad \text { on } \partial \Omega \text {, } \\
& \sigma_{e} \nabla u_{e} \cdot \nu=0 \quad \Leftrightarrow \quad \nabla u_{e} \cdot \nu=0 \quad \text { on } \partial \Omega \text {. }
\end{aligned}
$$

It is known due to [4] that (2.2) is biological reasonable.

First, we want to introduce the bidomain operator in a weak setting as well as the corresponding bidomain bilinear form. Therefore, we define $V_{a v}(\Omega):=\left\{u \in V: \int_{\Omega} u \mathrm{~d} x=0\right\}$. Following [3], we define the bilinear forms

$$
a_{i}(u, v):=\int_{\Omega} \sigma_{i} \nabla u \cdot \nabla v \mathrm{~d} x, \quad a_{e}(u, v):=\int_{\Omega} \sigma_{e} \nabla u \cdot \nabla v \mathrm{~d} x
$$

for all $(u, v) \in V_{a v} \times V_{a v}$. Due to (2.1) these bilinear forms are symmetric, continuous and uniformly elliptic on $V_{a v} \times V_{a v}$. Then, we define the weak operators $A_{i}$ and $A_{e}$ from $V_{a v}$ onto $V_{a v}^{\prime}$ by

$$
\left\langle A_{i} u, v\right\rangle:=a_{i}(u, v), \quad\left\langle A_{e} u, v\right\rangle:=a_{e}(u, v)
$$

for all $(u, v) \in V_{a v} \times V_{a v}$. Let $P_{a v}$ be the orthogonal projection from $V$ to $V_{a v}(\Omega)$, i.e., $P_{a v} u:=$ $u-\frac{1}{|\Omega|} \int_{\Omega} u \mathrm{~d} x$ and denote its transpose by $P_{a v}^{T}: V_{a v}^{\prime} \rightarrow V^{\prime}$. Now we are able to define the weak bidomain operator and the corresponding bidomain bilinear form as

$$
\begin{aligned}
A & =P_{a v}^{T} A_{i}\left(A_{i}+A_{e}\right)^{-1} A_{e} P_{a v}, \\
a(u, v) & =\langle A u, v\rangle
\end{aligned}
$$

for all $(u, v) \in V \times V$. We have the following lemma.

Lemma 2.1 ([3, Theorem 6$])$. The bidomain bilinear form $a(\cdot, \cdot)$ is symmetric, continuous and coercive on $V$,

$$
\begin{aligned}
\alpha\|u\|_{V}^{2} & \leq a(u, u)+\alpha\left\|_{u}\right\|_{H}^{2}, & \text { for all } u & \in V, \\
|a(u, v)| & \leq M\|u\|_{V}\|v\|_{V}, & \text { for all } u, v & \in V,
\end{aligned}
$$


for some constants $\alpha, M>0$. Furthermore, there exists an increasing sequence $0=\lambda_{0}<\cdots \leq \lambda_{i} \leq \cdots$ in $\mathbb{R}$ and an orthonormal Hilbert basis of $H$ of eigenvectors $\left(\psi_{i}\right)_{i \in \mathbb{N}}$ such that for all $i \in \mathbb{N}, \psi_{i} \in V$ and $v \in V$ it is $a\left(\psi_{i}, v\right)=\lambda_{i}\left(\psi_{i}, v\right)$.

We next define the strong bidomain operator in the $L^{q}$-setting for $1<q<\infty$. We will use the same notation as for the weak setting since it will be clear from the context whether we consider the weak or strong formulation. To this end, let $L_{a v}^{q}(\Omega):=\left\{u \in L^{q}(\Omega): \int_{\Omega} u \mathrm{~d} x=0\right\}$ and let $P_{a v}$ be the orthogonal projection from $L^{q}(\Omega)$ to $L_{a v}^{q}(\Omega)$, i.e., $P_{a v} u:=u-\frac{1}{|\Omega|} \int_{\Omega} u \mathrm{~d} x$. Then, we define the elliptic operators $A_{i}$ and $A_{e}$ by

$$
\begin{aligned}
A_{i, e} u & :=-\operatorname{div}\left(\sigma_{i, e} \nabla u\right), \\
D\left(A_{i, e}\right) & :=\left\{u \in W^{2, q}(\Omega) \cap L_{a v}^{q}(\Omega): \sigma_{i, e} \nabla u \cdot \nu=0 \text { a.e. on } \partial \Omega\right\} \subset L_{a v}^{q}(\Omega) .
\end{aligned}
$$

Here $A_{i, e}$ and $\sigma_{i, e}$ mean that either $A_{i}$ and $\sigma_{i}$ or $A_{e}$ and $\sigma_{e}$ are considered. Condition (2.2) implies that $D\left(A_{i}\right)=D\left(A_{e}\right)$. Hence, it is possible to define the sum $A_{i}+A_{e}$ with the domain $D\left(A_{i}\right)=D\left(A_{e}\right)$. Note that the inverse operator $\left(A_{i}+A_{e}\right)^{-1}$ on $L_{a v}^{q}(\Omega)$ is a bounded linear operator.

Following [11] we define the bidomain operator as follows. Let $\sigma_{i}$ and $\sigma_{e}$ satisfy Assumption C] Then the bidomain operator $A$ is defined as

$$
A=A_{i}\left(A_{i}+A_{e}\right)^{-1} A_{e} P_{a v}
$$

with domain

$$
D(A):=\left\{u \in W^{2, q}(\Omega): \nabla u \cdot \nu=0 \text { a.e. on } \partial \Omega\right\} .
$$

If we assume conservation of currents, i.e.,

$$
\int_{\Omega}\left(s_{i}(t)+s_{e}(t)\right) \mathrm{d} x=0, \quad t \geq 0
$$

and moreover $\int_{\Omega} u_{e} \mathrm{~d} x=0$, the bidomain equations (BDE) may be equivalently rewritten as an evolution equation $[3,11]$ of the form

$$
\left\{\begin{array}{rlrl}
\partial_{t} u+A u+f(u, w) & =s, & & \text { in }(0, \infty), \\
\partial_{t} w+g(u, w) & =0, & & \text { in }(0, \infty), \\
u(0)=u_{0}, & w(0)=w_{0}, &
\end{array}\right.
$$

where

$$
s:=s_{i}-A_{i}\left(A_{i}+A_{e}\right)^{-1}\left(s_{i}+s_{e}\right)
$$

is the modified source term. The functions $u_{e}$ and $u_{i}$ can be recovered from $u$ by virtue of the following relations

$$
\begin{aligned}
& u_{e}=\left(A_{i}+A_{e}\right)^{-1}\left\{\left(s_{i}+s_{e}\right)-A_{i} P_{a v} u\right\}, \\
& u_{i}=u+u_{e} .
\end{aligned}
$$

\section{WEAK TIME-PERIODIC SOLUTIONS}

In this section, we show the existence of weak time-periodic solutions by using a Galerkin approximation. We consider the bidomain equations under some growth conditions on $f$ and $g$ which contain the nonlinearities introduced in Section 1 .

We use the abstract form

(PABDE)

$$
\left\{\begin{array}{clrl}
u^{\prime}+A u+f(u, w) & =s, & & \text { in } \mathbb{T} \times \Omega, \\
w^{\prime}+g(u, w) & =0, & & \text { in } \mathbb{T} \times \Omega, \\
u(t+T, x)=u(t, x), & w(t+T)=w(t, x), &
\end{array}\right.
$$


where $s$ is a $T$-periodic function for some $T>0$ and $\mathbb{T}:=\mathbb{R} / T \mathbb{Z}$ denotes the time torus. We assume that the nonlinear terms $f$ and $g$ satisfy the following conditions.

Assumption N. Let $p>1$ be the number so that the Sobolev embedding $V \subset L^{p}(\Omega)$ holds. In other words, $2 \leq p$ if $d=2$; or $2 \leq p \leq 6$ if $d=3$. The nonlinear terms $f, g: \mathbb{R} \times \mathbb{R} \rightarrow \mathbb{R}$ are of the form

$$
\begin{aligned}
& f(u, w)=f_{1}(u)+f_{2}(u) w, \\
& g(u, w)=g_{1}(u)+g_{2} w,
\end{aligned}
$$

where $g_{2} \in \mathbb{R}$ and $f_{1}, f_{2}, g_{1}: \mathbb{R} \rightarrow \mathbb{R}$ are continuous functions. The functions are assumed to satisfy that there exist constants $C_{0} \in \mathbb{R}, C_{i}>0(i=1, \ldots, 5)$ and $r>0$ such that

$$
\begin{aligned}
C_{0}+C_{1}|u|^{p}+C_{2}|w|^{2} & \leq r f(u, w) u+g(u, w) w \\
\left|f_{1}(u)\right| & \leq C_{3}\left(1+|u|^{p-1}\right) \\
\left|f_{2}(u)\right| & \leq C_{4}\left(1+|u|^{p / 2-1}\right) \\
\left|g_{1}(u)\right| & \leq C_{5}\left(1+|u|^{p / 2}\right)
\end{aligned}
$$

for all $u, w \in \mathbb{R}$.

This assumption is a modified version of the assumption used in [3]. Note that the three models introduced in Section 1 hold the assumption as $p=4$. We shall check it in the appendix. We see that the following inequality holds: for any $(u, w) \in L^{p}(\Omega) \times H$, we have

$$
\begin{aligned}
\|f(u, w)\|_{p^{\prime}}^{p^{\prime}} & \leq C_{6}\left(1+\|u\|_{p}^{p}+\|w\|_{H}^{2}\right) \\
\|g(u, w)\|_{H}^{2} & \leq C_{7}\left(1+\|u\|_{p}^{p}+\|w\|_{H}^{2}\right)
\end{aligned}
$$

for some $C_{i}>0(i=6,7)$ depending on $p$ and $C_{3}, \ldots, C_{5}$, where $p^{\prime}$ is the Hölder conjugate exponent, i.e., $1 / p+1 / p^{\prime}=1$. In particular, $f(u, w) \in L^{p^{\prime}}(Q)$ and $g(u, w) \in L^{2}(Q)$ for all $u \in L^{p}(Q), w \in L^{2}(Q)$. See in [3, Lemma 25].

Under this assumption, weak time-periodic solutions for (PABDE) are defined as follows.

Definition 3.1. Let $T>0, s \in L^{2}\left(\mathbb{T} ; V^{\prime}\right)$. Suppose that the Assumption $\mathbb{N}$ holds. Then a pair of $(u, w)$ of $u: \mathbb{T} \times \Omega \rightarrow \mathbb{R}, w: \mathbb{T} \times \Omega \rightarrow \mathbb{R}$ is called a weak $T$-periodic solution to (PABDE) if

(i) $u \in C_{w}(\mathbb{T} ; H) \cap L^{2}(\mathbb{T} ; V) \cap L^{p}(Q), w \in C_{w}(\mathbb{T} ; H)$,

(ii) For all $\varphi_{1} \in W^{1,2}(0, T ; H) \cap L^{2}(0, T ; V) \cap L^{p}(Q)$ and all $\varphi_{2} \in W^{1,2}(0, T ; H)$,

$$
\begin{aligned}
\int_{0}^{t}\left\{\left(u, \partial_{t} \varphi_{1}\right)-a\left(u, \varphi_{1}\right)-{ }_{p^{\prime}}\left\langle f(u, w), \varphi_{1}\right\rangle_{p}\right\} \mathrm{d} \tau & =-\int_{0}^{t} V^{\prime}\left\langle s, \varphi_{1}\right\rangle_{V} \mathrm{~d} \tau+\left(u(t), \varphi_{1}(t)\right)-\left(u(0), \varphi_{1}(0)\right), \\
\int_{0}^{t}\left\{\left(w, \partial_{t} \varphi_{2}\right)-\left(g(u, w), \varphi_{2}\right)\right\} \mathrm{d} \tau & =\left(w(t), \varphi_{2}(t)\right)-\left(w(0), \varphi_{2}(0)\right),
\end{aligned}
$$

for all $t \in(0, T)$. Here $(\cdot, \cdot)$ denotes the $L^{2}$-inner product.

A weak $T$-periodic solution $(u, w)$ is called strong if, in addition to above, it holds

$$
u \in W^{1,2}(\mathbb{T} ; H) \cap L^{2}\left(\mathbb{T} ; H^{2}(\Omega)\right), w \in W^{1,2}(\mathbb{T} ; H) .
$$

The result on existence of weak time-periodic solutions reads as follows.

Theorem 3.2. Let $T>0$. For every $T$-periodic function $s \in L^{2}\left(\mathbb{T} ; V^{\prime}\right)$ there exists at least one weak $T$-periodic solution $(u, w)$ to (PABDE).

Proof. Let $\left\{\psi_{i}\right\}_{i=0}^{\infty} \subset V$ be the orthonormal basis of eigenvectors of the bidomain bilinear form $a$ in $H$ and let $\left\{\lambda_{i}\right\}_{i=0}^{\infty} \subset \mathbb{R}_{\geq 0}$ be the corresponding eigenvalues, i.e., $a\left(U, \psi_{i}\right)=\lambda_{i}\left(U, \psi_{i}\right)_{L^{2}(\Omega)}$ for all $U \in V, i=0,1, \ldots$ Let

$$
u_{k}(t, x):=\sum_{i=0}^{k} \alpha_{k i}(t) \psi_{i}(x)
$$




$$
w_{k}(t, x):=\sum_{i=0}^{k} \beta_{k i}(t) \psi_{i}(x),
$$

with $\alpha_{k}(t)=\left\{\alpha_{k j}(t)\right\}_{j=0}^{k}, \beta_{k}(t)=\left\{\beta_{k j}(t)\right\}_{j=0}^{k}$, which are the solutions of the system of the ordinary differential equations

$$
\left\{\begin{aligned}
\frac{\mathrm{d}}{\mathrm{d} t} \alpha_{k j} & =-\alpha_{k j} \lambda_{j}-\int_{\Omega} f\left(u_{k}, w_{k}\right) \psi_{j} \mathrm{~d} x+V^{\prime}\left\langle s(t), \psi_{j}\right\rangle_{V}, \\
\frac{\mathrm{d}}{\mathrm{d} t} \beta_{k j} & =-\int_{\Omega} g\left(u_{k}, w_{k}\right) \psi_{j} \mathrm{~d} x, \\
\alpha_{k j}(0) & =a_{j} \\
\beta_{k j}(0) & =b_{j},
\end{aligned}\right.
$$

for $j=0,1, \ldots, k$. The initial data $\mathbf{a}_{k}=\left\{a_{j}\right\}_{j=1}^{k}$ and $\mathbf{b}_{k}=\left\{b_{j}\right\}_{j=1}^{k}$ are fixed later. By the standard theory of ordinary differential equations, this system admits a unique solution $\left(\alpha_{k}, \beta_{k}\right) \subset\left(W^{1,2}\left(0, T_{k}\right)\right)^{2(k+1)}$ on some interval $\left(0, T_{k}\right)$. It is either $\left|\alpha_{k}(t)\right|+\left|\beta_{k}(t)\right| \rightarrow \infty$ as $t \nearrow T_{k}$ or we can take any finite time $T_{k}$. In the following, it is shown that $\left|\alpha_{k}(t)\right|+\left|\beta_{k}(t)\right| \rightarrow \infty$ as $t \nearrow T_{k}$ does not occur by using a priori estimates. To this end, multiplying the first equation of (3.7) with $r \cdot \alpha_{k j}$, the second equation with $\beta_{k j}$, and summing over $j$ yield

$$
\begin{aligned}
& \frac{1}{2} \frac{\mathrm{d}}{\mathrm{d} t}\left(r\left\|u_{k}(t)\right\|_{H}^{2}+\left\|w_{k}(t)\right\|_{H}^{2}\right)+r a\left(u_{k}(t), u_{k}(t)\right)+\int_{\Omega} r f\left(u_{k}(t), w_{k}(t)\right) u_{k}(t)+g\left(u_{k}(t), w_{k}(t)\right) w_{k}(t) \mathrm{d} x \\
& =r_{V^{\prime}}\left\langle s(t), u_{k}(t)\right\rangle_{V} .
\end{aligned}
$$

We recall that the bidomain bilinear form $a$ has the coercivity of the form

$$
\alpha\|U\|_{V}^{2} \leq a(U, U)+\alpha\|U\|_{H}^{2}
$$

for all $U \in V$ and for some constant $\alpha>0$, see [3]. By the coercivity of $a$, the Assumption $\mathbb{N}$ and Young's inequality, it is

$$
\begin{aligned}
& \frac{\mathrm{d}}{\mathrm{d} t}\left(r\left\|u_{k}(t)\right\|_{H}^{2}+\left\|w_{k}(t)\right\|_{H}^{2}\right)+C_{11}\left\|u_{k}(t)\right\|_{V}^{2}+C_{12}\left\|u_{k}(t)\right\|_{p}^{p}-C_{13}\left\|u_{k}(t)\right\|_{H}^{2}+C_{14}\left\|w_{k}(t)\right\|_{H}^{2} \\
\leq & C_{15}\|s(t)\|_{V^{\prime}}^{2}+C_{16},
\end{aligned}
$$

for some constants $C_{1 i}=C_{1 i}\left(r, \alpha, C_{j}\right)>0(i=1, \ldots, 6, j=0, \ldots, 2)$; we emphasize that all constants $C_{1 i}$ are independent of $k$. We use the estimate

$$
C_{17}\left\|u_{k}(t)\right\|_{p}^{p}-C_{18} \leq C_{12}\left\|u_{k}(t)\right\|_{p}^{p}-C_{13}\left\|u_{k}(t)\right\|_{2}^{2}
$$

for some $C_{17}, C_{18}>0$ since $2<p<\infty$. Therefore, we have the following estimate

$$
\begin{aligned}
& \frac{\mathrm{d}}{\mathrm{d} t}\left(r\left\|u_{k}(t)\right\|_{H}^{2}+\left\|w_{k}(t)\right\|_{H}^{2}\right)+C_{21}\left(r\left\|u_{k}(t)\right\|_{V}^{2}+\left\|u_{k}(t)\right\|_{p}^{p}+\left\|w_{k}(t)\right\|_{H}^{2}\right) \\
\leq & C_{22}\|s(t)\|_{V^{\prime}}^{2}+C_{23},
\end{aligned}
$$

for some constants $C_{2 i}>0(i=1,2,3)$.

Then, we apply Gronwall's inequality for the inequality (3.8), then

$$
\begin{aligned}
& r\left\|u_{k}(t)\right\|_{H}^{2}+\left\|w_{k}(t)\right\|_{H}^{2} \\
\leq & \mathrm{e}^{-C_{21} t}\left(r\left\|\mathbf{a}_{k}\right\|_{H}^{2}+\left\|\mathbf{b}_{k}\right\|_{H}^{2}\right)+\int_{0}^{t} \mathrm{e}^{-C_{21}(t-\tau)}\left(C_{22}\|s(\tau)\|_{V^{\prime}}^{2}+C_{23}\right) \mathrm{d} \tau .
\end{aligned}
$$

Since $\left\|u_{k}(t)\right\|_{H}^{2}=\left|\alpha_{k}(t)\right|^{2}$ and $\left\|w_{k}(t)\right\|_{H}^{2}=\left|\beta_{k}(t)\right|^{2}$, this implies $T_{k}$ does not blow up at any finite time. We consider the Poincaré map

$$
\begin{array}{r}
\mathcal{S}: \mathbb{R}^{k+1} \times \mathbb{R}^{k+1} \rightarrow \mathbb{R}^{k+1} \times \mathbb{R}^{k+1}, \\
\mathcal{S}\left(\mathbf{a}_{k}, \mathbf{b}_{k}\right):=\left(\alpha_{k}(T), \beta_{k}(T)\right) .
\end{array}
$$


We define

$$
\mathbb{B}_{R}:=\left\{\left(\mathbf{a}_{k}, \mathbf{b}_{k}\right)=\left(\left\{a_{j}\right\}_{j=0}^{k},\left\{b_{j}\right\}_{j=0}^{k}\right) \in \mathbb{R}^{k+1} \times \mathbb{R}^{k+1} \mid r\left(\sum_{j=0}^{k}\left|a_{j}\right|^{2}\right)^{1 / 2}+\left(\sum_{j=0}^{k}\left|b_{j}\right|^{2}\right)^{1 / 2} \leq R\right\}
$$

with

$$
R^{2}=\frac{\int_{0}^{T} \mathrm{e}^{-C_{21}(T-\tau)}\left(C_{22}\|s(\tau)\|_{V^{\prime}}^{2}+C_{23}\right) \mathrm{d} \tau}{1-\mathrm{e}^{-C_{21} T}} .
$$

Then, it follows that $\mathcal{S}$ maps $\mathbb{B}_{R}$ into itself from (3.9). Since $\mathcal{S}$ is also continuous, by Brouwer's fixed point theorem we conclude that $\mathcal{S}$ admits a fixed point $\left(\overline{\mathbf{a}}_{k}, \overline{\mathbf{b}}_{k}\right)=\mathcal{S}\left(\overline{\mathbf{a}}_{k}, \overline{\mathbf{b}}_{k}\right)$ in $\mathbb{B}_{R}$ for all $k \in \mathbb{N}$.

In the following, we denote by $u_{k}$ and $w_{k}$ the functions defined in (3.5) and (3.6) respectively, corresponding to the solutions $\alpha_{k}, \beta_{k}$ of (3.7) with initial values $\overline{\mathbf{a}}_{k}, \overline{\mathbf{b}}_{k}$. Then, $u_{k}(0, x)=u_{k}(T, x)$ and $w_{k}(0, x)=w_{k}(T, x)$. Moreover, we see $u_{k}(t+T, x)=u_{k}(t, x)$ and $w_{k}(t+T, x)=w_{k}(t, x)$ for all $t \in \mathbb{R}$ by periodically expansion. In the next step, we would like to pass to the limit $k \rightarrow \infty$ and show the existence of a weak solution to the original problem (ABDE). To do so, we consider the uniform boundedness. For the inequality (3.9), we take the supremum from $t=0$ to $t=T$, then by using (3.10)

$$
\left\|u_{k}\right\|_{L^{\infty}(0, T ; H)}+\left\|w_{k}\right\|_{L^{\infty}(0, T ; H)} \leq C_{31}\|s\|_{L^{2}\left(0, T ; V^{\prime}\right)}+C_{32},
$$

for some $C_{3 i}>0(i=1,2)$. Moreover, for the inequality (3.8), integrate from $t=0$ to $t=T$ to get

$$
\left\|u_{k}\right\|_{L^{2}(0, T ; V)}^{2}+\left\|u_{k}\right\|_{L^{p}(Q)}^{p}+\left\|w_{k}\right\|_{L^{2}(0, T ; H)}^{2} \leq C_{41}\|s\|_{L^{2}\left(0, T ; V^{\prime}\right)}^{2}+C_{42} .
$$

for some $C_{4 i}>0(i=1,2)$. This implies that there are sub-sequences of $\left\{u_{k}\right\}_{k=1}^{\infty}$ and $\left\{w_{k}\right\}_{k=1}^{\infty}$, for convenience still denoted by $\left\{u_{k}\right\}_{k=1}^{\infty}$ and $\left\{w_{k}\right\}_{k=1}^{\infty}$, that converges to $u$ weakly in $L^{2}(0, T ; V) \cap L^{p}(Q)$ and converges to $w$ weakly in $L^{2}(0, T ; H)$.

By construction of the function $u_{k}$ and $w_{k}$,

$$
\begin{aligned}
\left(\partial_{t} u_{k}(t), \psi_{\ell}\right)+a\left(u_{k}(t), \psi_{\ell}\right)+{ }_{p^{\prime}}\left\langle f\left(u_{k}, w_{k}\right), \psi_{\ell}\right\rangle_{p} & ={ }_{V^{\prime}}\left\langle s(t), \psi_{\ell}\right\rangle_{V} \\
\left(\partial_{t} w_{k}(t), \psi_{\ell}\right)+\left(g\left(u_{k}, w_{k}\right), \psi_{\ell}\right) & =0
\end{aligned}
$$

for all $\ell=0, \ldots, k$. Integrate from $t_{0}$ to $t_{1}\left(0 \leq t_{0} \leq t_{1} \leq T\right)$, then we get

$$
\begin{aligned}
& \left|\left(u_{k}\left(t_{1}\right), \psi_{\ell}\right)-\left(u_{k}\left(t_{0}\right), \psi_{\ell}\right)\right| \\
= & \left|\int_{t_{0}}^{t_{1}}-a\left(u_{k}, \psi_{\ell}\right)-{ }_{p^{\prime}}\left\langle f\left(u_{k}, w_{k}\right), \psi_{\ell}\right\rangle_{p}+{ }_{V^{\prime}}\left\langle s, \psi_{\ell}\right\rangle_{V} \mathrm{~d} \tau\right| \\
\leq & \left\|u_{k}\right\|_{L^{2}(0, T ; V)}\left\|\psi_{\ell}\right\|_{L^{2}(0, T ; V)}+\left\|f\left(u_{k}, w_{k}\right)\right\|_{L^{p^{\prime}}(Q)}\left\|\psi_{\ell}\right\|_{L^{p}(Q)}+\|s\|_{L^{2}\left(t_{0}, t_{1} ; V^{\prime}\right)}\left\|\psi_{\ell}\right\|_{L^{2}(0, T ; V)} \\
\leq & C\left(\left|t_{1}-t_{0}\right|^{1 / 2}+\left|t_{1}-t_{0}\right|^{1 / p}+\|s\|_{L^{2}\left(t_{0}, t_{1} ; V^{\prime}\right)}\right) \\
& \left|\left(w_{k}\left(t_{1}\right), \psi_{\ell}\right)-\left(w_{k}\left(t_{0}\right), \psi_{\ell}\right)\right| \\
= & \left|\int_{t_{0}}^{t_{1}}-\left(g\left(u_{k}, w_{k}\right), \psi_{\ell}\right) \mathrm{d} \tau\right| \\
= & \left\|g\left(u_{k}, w_{k}\right)\right\|_{L^{2}(Q)}\|\psi\|_{L^{2}(Q)} \\
\leq & C\left|t_{1}-t_{0}\right|^{1 / 2}
\end{aligned}
$$

for some $C=C\left(s, \psi_{\ell}\right)$ independent of $t_{0}, t_{1}$ and $k$, where we use the inequality (3.11) and the embedding assumption $\left(\psi_{\ell} \in\right) V \subset L^{p}(\Omega)$. Therefore, it follows that for any $\varepsilon>0$ there is a $\delta>0$ with

$$
\left|\left(u_{k}\left(t_{1}\right), \psi_{\ell}\right)-\left(u_{k}\left(t_{0}\right), \psi_{\ell}\right)\right|+\left|\left(w_{k}\left(t_{1}\right), \psi_{\ell}\right)-\left(w_{k}\left(t_{0}\right), \psi_{\ell}\right)\right|<\varepsilon \quad \text { if }\left|t_{1}-t_{0}\right| \leq \delta, k=1,2, \ldots
$$

This means the families $\left\{\left(u_{k}(t), \psi_{\ell}\right)\right\}_{k=1}^{\infty}$ and $\left\{\left(w_{k}(t), \psi_{\ell}\right)\right\}_{k=1}^{\infty}$ are equicontinuous. Since $\left\{\left(u_{k}, \psi_{\ell}\right)\right\}_{k=1}^{\infty}$ and $\left\{\left(w_{k}, \psi_{\ell}\right)\right\}_{k=1}^{\infty}$ are uniform bounded in $k$, from Ascoli-Arzela's theorem, it follows that the subsequences $\left\{\left(u_{k}(t), \psi_{\ell}\right)\right\}_{k=1}^{\infty}$ and $\left\{\left(w_{k}(t), \psi_{\ell}\right)\right\}_{k=1}^{\infty}$ converge uniformly to continuous functions $\left(u(t), \psi_{\ell}\right)$ and 
$\left(w(t), \psi_{\ell}\right)$ for each fixed $\ell$. By the Cantor diagonalization argument and a density argument, this convergence can be generalized that for each $\psi \in H,\left\{\left(u_{k}(t), \psi\right)\right\}_{k=1}^{\infty}$ and $\left\{\left(w_{k}(t), \psi\right)\right\}_{k=1}^{\infty}$ converge uniformly to continuous functions $(u(t), \psi)$ and $(w(t), \psi)$. Therefore, we have $u \in C_{w}(\mathbb{T} ; H)$ and $w \in C_{w}(\mathbb{T} ; H)$.

It remains to show the weak convergence of the nonlinear terms $f\left(u_{k}, w_{k}\right), g\left(u_{k}, w_{k}\right)$. We first prove $u_{k} \rightarrow u$ in $L^{2}(Q)$. To do so, we use Friedrich's inequality, which states that for any $\varepsilon>0$, there exists $J \in \mathbb{N}$ and $\phi_{1}, \phi_{2}, \ldots, \phi_{J} \in H$ such that for all $U \in V$, the following inequality holds

$$
\|U\|_{H}^{2} \leq \sum_{j=1}^{J}\left|\int_{\Omega} U \phi_{j} \mathrm{~d} x\right|^{2}+\varepsilon\|\nabla U\|_{H}^{2} .
$$

For Friedrich's inequality, see e.g. [10]. This inequality with $U=u_{k}-u$, the uniform boundedness of $\left\{u_{k}\right\}_{k=1}^{\infty} \subset L^{2}(0, T ; V)$, and $u_{k} \rightarrow u$ in $C_{w}(\mathbb{T} ; H)$ implies that $u_{k} \rightarrow u$ in $L^{2}(Q)$. Since we have $u_{k} \rightarrow u$ a.e. in $Q$ and $f_{1}, f_{2}, g_{1}$ are continuous, $f_{1}\left(u_{k}\right) \rightarrow f_{1}(u), f_{2}\left(u_{k}\right) \rightarrow f_{2}(u), g_{1}\left(u_{k}\right) \rightarrow g(u)$ a.e. in $Q$ are satisfied. We shall show uniform boundedness in $L^{p^{\prime}}(Q)$ for $f\left(u_{k}, w_{k}\right)$ and uniform boundedness in $L^{2}(Q)$ for $g\left(u_{k}, w_{k}\right)$, which implies $f\left(u_{k}, w_{k}\right) \rightarrow f(u, w)$ weakly in $L^{p^{\prime}}(Q)$ and $g\left(u_{k}, w_{k}\right) \rightarrow g(u, w)$ weakly in $L^{2}(Q)$. Fortunately, under the Assumption $\mathbb{N}$, it has already been proved in [3, p.477]. Since the functions $u_{k}, w_{k}$ satisfy that for all $\varphi_{1} \in H^{1}(0, T ; H) \cap L^{2}(0, T ; V) \cap L^{p}(Q)$ and all $\varphi_{2} \in W^{1,2}(0, T ; H)$,

$$
\begin{aligned}
\int_{0}^{t}\left\{\left(u_{k}, \partial_{t} \varphi_{1}\right)-a\left(u_{k}, \varphi_{1}\right)-{ }_{p^{\prime}}\left\langle f\left(u_{k}, w_{k}\right), \varphi_{1}\right\rangle_{p}\right\} \mathrm{d} \tau & =-\int_{0}^{t} V^{\prime}\left\langle s, \varphi_{1}\right\rangle_{V} \mathrm{~d} \tau+\left(u_{k}(t), \varphi_{1}(t)\right)-\left(u_{k}(0), \varphi_{1}(0)\right), \\
\int_{0}^{t}\left\{\left(w_{k}, \partial_{t} \varphi_{2}\right)-\left(g\left(u_{k}, w_{k}\right), \varphi_{2}\right)\right\} \mathrm{d} \tau & =\left(w_{k}(t), \varphi_{2}(t)\right)-\left(w_{k}(0), \varphi_{2}(0)\right),
\end{aligned}
$$

for all $t \in(0, T)$, combining above discussions about the weak convergence, we show the existence of a weak $T$-periodic solution.

\section{Regularity of WEAK PERIOdic SOlution}

In this section, we shall show that for the FitzHugh-Nagumo nonlinearities introduced in Section 1 the weak time-periodic solution constructed in the previous section is actually a strong solution. In order to do so, we first review the global strong well-posedness result by Colli Franzone and Savaré [7]. After that, we use a weak-strong uniqueness argument to show the existence of a strong time-periodic solution for (PABDE) with FitzHugh-Nagumo nonlinearities. In [7], they considered the initial boundary value problem for the bidomain equations of the form

(BDE II)

$$
\left\{\begin{aligned}
\partial_{t} u-\operatorname{div}\left(\sigma_{i} \nabla u_{i}\right)+f(u)+\theta w & =s_{i}, & & \text { in }(0, \infty) \times \Omega, \\
\partial_{t} u+\operatorname{div}\left(\sigma_{e} \nabla u_{e}\right)+f(u)+\theta w & =-s_{e}, & & \text { in }(0, \infty) \times \Omega, \\
u & =u_{i}-u_{e}, & & \text { in }(0, \infty) \times \Omega, \\
\partial_{t} w+\gamma w-\eta u & =0, & & \text { in }(0, \infty) \times \Omega, \\
\sigma_{i} \nabla u_{i} \cdot \nu=g_{i}, \sigma_{e} \nabla u_{e} \cdot \nu & =g_{e}, & & \text { in }(0, \infty) \times \partial \Omega, \\
u(0)=u_{0}, & w(0)=w_{0}, & & \text { in } \Omega,
\end{aligned}\right.
$$

with $\theta, \gamma, \eta>0$.

They regarded the bidomain equation as the degenerate variational formulation of the form

$$
\left\{\begin{aligned}
(B u)^{\prime}+A u+\mathcal{F} u & =L \quad t \in(0, T) \\
(B u)(0) & =\ell^{0}
\end{aligned}\right.
$$

and constructed the global weak formulation and their regularity.

Let $\Omega$ be a Lipschitz domain of $\mathbb{R}^{d}, \Gamma:=\partial \Omega$, and the measurable function $\sigma_{i, e}: \bar{\Omega} \rightarrow \mathbb{R}^{d \times d}$ satisfy the uniform ellipticity condition. Assume the nonlinear term $f$ is a continuous function with

$$
f(0)=0, \exists \lambda_{f} \geq 0: \frac{f(x)-f(y)}{x-y} \geq-\lambda_{f}, \forall x, y \in \mathbb{R}, \text { with } x \neq y .
$$


Their result is as follows.

Theorem 4.1 (Franzone-Savaré '02 [7]). Let us assume $s_{i, e} \in L^{2}(0, \mathcal{T} ; H), g_{i, e} \in W^{1,1}\left(0, \mathcal{T} ; H^{-1 / 2}(\Gamma)\right)$ satisfy $s_{i}+s_{e} \in W^{1,1}(0, \mathcal{T} ; H)$ and the compatibility condition

$$
\int_{\Omega}\left(s_{i}+s_{e}\right) \mathrm{d} x+{ }_{H^{-1 / 2}(\Gamma)}\left\langle g_{i}+g_{e}, 1\right\rangle_{H^{1 / 2}(\Gamma)}=0 .
$$

Then for any initial data $u_{0}, w_{0} \in H$, there uniquely exist a couple

and

$$
u_{i, e} \in L^{2}(0, \mathcal{T} ; V), \int_{\Omega} u_{e}=0 \text { a.e } t
$$

$$
\begin{aligned}
& u \in C([0, \mathcal{T}] ; H) \cap L^{2}(0, \mathcal{T} ; V), \partial_{t} u \in L_{l o c}^{2}(0, \mathcal{T} ; H), \\
& f(u(t)) \in L^{1}(\Omega) \cap V^{\prime} \text { a.e. } t \in(0, \mathcal{T}), \\
& w, \partial_{t} w \in C([0, \mathcal{T}] ; H),
\end{aligned}
$$

which solves the bidomain equation in the sense of

$$
\begin{aligned}
& \int_{\Omega}\left(\partial_{t} u \hat{u}+\frac{\theta}{\eta} \partial_{t} w \hat{w}\right) \mathrm{d} x+\int_{\Omega} f(u) \hat{u} \mathrm{~d} x+\sum_{i, e} \int_{\Omega} \sigma_{i, e} \nabla u_{i, e} \cdot \nabla \hat{u}_{i, e} \mathrm{~d} x+\frac{\theta \gamma}{\eta} \int_{\Omega} w \hat{w} \mathrm{~d} x+\theta \int_{\Omega}(w \hat{u}-u \hat{w}) \mathrm{d} x \\
& =\sum_{i, e} \int_{\Omega} s_{i, e} \hat{u}_{i, e} \mathrm{~d} x+\sum_{i, e} H^{-1 / 2(\Gamma)}\left\langle g_{i, e}, \hat{u}_{i, e}\right\rangle_{H^{1 / 2}(\Gamma)}, \\
& \int_{\Omega}\left(u(0) \hat{u}+\frac{\theta}{\eta} w(0) \hat{w}\right) \mathrm{d} x=\int_{\Omega}\left(u_{0} \hat{u}+\frac{\theta}{\eta} w_{0} \hat{w}\right) \mathrm{d} x,
\end{aligned}
$$

for a.e. $t \in(0, \mathcal{T})$ and all $\hat{u}_{i, e} \in V \times V$ with $\int_{\Omega} \hat{u}_{e} \mathrm{~d} x=0$ and $\hat{u}=\hat{u}_{i}-\hat{u}_{e}$ and $\hat{w} \in H$.

Moreover if $u_{0} \in V, u_{0} f\left(u_{0}\right) \in L^{1}(\Omega)$, then

$$
u_{i, e} \in C([0, \mathcal{T}] ; V), \partial_{t} u \in L^{2}(0, \mathcal{T} ; H), w \in C([0, \mathcal{T}] ; V) .
$$

Furthermore they derived the regularity results.

Proposition 4.2. In addition to the assumption in the theorem, suppose that $d=3$, and the nonlinear term $f$ has a cubic growth at infinity, i.e.,

$$
0<\liminf _{|r| \rightarrow \infty} \frac{f(r)}{r^{3}} \leq \limsup _{|r| \rightarrow \infty} \frac{f(r)}{r^{3}}<+\infty .
$$

Then the bidomain equation admits a unique strong solution $u_{i, e}, u, w$. Moreover, it satisfies

$$
-\operatorname{div}\left(\sigma_{i, e} \nabla u_{i, e}\right) \in L^{2}(0, \mathcal{T} ; H)
$$

Remark 4.3. Let $\Omega$ be of class $C^{1,1}, \sigma_{i, e}$ be Lipschitz in $\Omega$ and $g_{i, e} \in L^{2}\left(0, \mathcal{T} ; H^{1 / 2}(\Gamma)\right)$. Then by the standard regularity theorem, we see

$$
u_{i, e} \in L^{2}\left(0, \mathcal{T} ; H^{2}(\Omega)\right) .
$$

Remark 4.4. If we look at the function $f$ of the FitzHugh-Nagumo nonlinearity introduced in Section! as $f(u, w)=f(u)+w=u(u-a)(u-1)+w$, then the function $f(u)$ satisfies the assumptions for the nonlinearity in Proposition 4.2 as well as Assumption (4.1).

Now, we combine the results from the previous sections to obtain a strong time-periodic solution for the bidomain equations with FitzHugh-Nagumo type nonlinearities subject to arbitrary large forces. We would like to identify our weak time-periodic solution $(v, z)$ constructed in Section 3 with a strong solution $(u, w)$ to the initial value problem with initial data $v\left(t_{0}\right), z\left(t_{0}\right)$ for some $t_{0}>0$ satisfying $v\left(t_{0}\right) \in V$ and $f\left(v\left(t_{0}\right)\right) v\left(t_{0}\right) \in L^{1}(\Omega)$. Since $f$ has cubic growth, i.e., $p=4$ in the Assumption (N), $v \in L^{4}(Q)$ derives the existence of $t_{0}$. So we can use the theorem by Colli-Franzone and Savaré for the 
global strong solution with the initial values $v\left(t_{0}\right), z\left(t_{0}\right)$. Finally, we show that the weak solution $(v, z)$ coincides with the strong solution $(u, w)$ and therefore obtain the existence of a strong time-periodic solution. We follow the approach given in [12]

To be more precise, for given $T$-time-periodic functions $s_{i, e} \in L^{2}(0, \mathcal{T} ; H)$ with $s_{i}+s_{e} \in W^{1,1}(0, \mathcal{T} ; H)$ and $\int_{\Omega}\left(s_{i}+s_{e}\right) \mathrm{d} x=0$ for a.e. $t$, let $(v, z)$ be a weak $T$-time-periodic solution of (PABDE) for $s=s_{i}-A_{i}\left(A_{i}+A_{e}\right)^{-1}\left(s_{i}+s_{e}\right)\left(\in L^{2}(\mathbb{T} ; H)\right)$ corresponding to Theorem 3.2. We take $t_{0}$ such that $v\left(t_{0}\right) \in V$ and $v\left(t_{0}\right) f\left(v\left(t_{0}\right)\right) \in L^{1}(\Omega)$. Since $(v, z)$ is a weak $T$-time-periodic solution, it satisfies that for all $\varphi_{1} \in W^{1,2}\left(t_{0}, \mathcal{T} ; H\right) \cap L^{2}\left(t_{0}, \mathcal{T} ; V\right) \cap L^{4}(Q)$ and all $\varphi_{2} \in W^{1,2}\left(t_{0}, \mathcal{T} ; H\right)$

$$
\begin{aligned}
\int_{t_{0}}^{t}\left\{\left(v, \partial_{t} \varphi_{1}\right)-a\left(v, \varphi_{1}\right)-\left(f(v, z), \varphi_{1}\right)\right\} \mathrm{d} \tau & =-\int_{t_{0}}^{t}\left(s(\tau), \varphi_{1}(\tau)\right) \mathrm{d} \tau+\left(v(t), \varphi_{1}(t)\right)-\left(v\left(t_{0}\right), \varphi_{1}\left(t_{0}\right)\right), \\
4.3) \quad \int_{t_{0}}^{t}\left\{\left(z, \partial_{t} \varphi_{2}\right)-\left(g(w, z), \varphi_{2}\right)\right\} \mathrm{d} \tau & =\left(z(t), \varphi_{2}(t)\right)-\left(z\left(t_{0}\right), \varphi_{2}\left(t_{0}\right)\right),
\end{aligned}
$$

for all $t \in\left(t_{0}, \mathcal{T}\right)$, and $(v, z)$ satisfies the following strong energy inequality:

$$
\left(\|v(t)\|_{H}^{2}+\|z(t)\|_{H}^{2}\right)+2 \int_{t_{0}}^{t} a(v(\tau), v(\tau)) \mathrm{d} \tau+2 \int_{t_{0}}^{t} \int_{\Omega} f(v(\tau), z(\tau)) v(\tau)+g(v(\tau), z(\tau)) z(\tau) \mathrm{d} x \mathrm{~d} \tau
$$

$$
\leq\left\|v\left(t_{0}\right)\right\|_{H}^{2}+\left\|z\left(t_{0}\right)\right\|_{H}^{2}+2 \int_{t_{0}}^{t}(s(\tau), v(\tau)) \mathrm{d} \tau,
$$

for all $t \in\left[t_{0}, \mathcal{T}\right]$.

We next consider the unique global strong solution $(u, w) \in\left(W^{1,2}\left(t_{0}, \mathcal{T} ; H\right) \cap L^{2}\left(0, \mathcal{T} ; H^{2}(\Omega)\right)\right) \times$ $C^{1}([0, \mathcal{T}] ; H)$ corresponding to the initial-boundary value problem for the bidomain equation with initial value $\left(v\left(t_{0}\right), z\left(t_{0}\right)\right)$ and $T$-periodic right-hand side $s_{i, e}$ and $g_{i, e}=0$. In the following, we show that the weak solution $(v, z)$ agrees with the strong solution $(u, w)$.

Since $(u, w)$ is a strong solution, it satisfies that for all $\mathcal{T}>t_{0}$ and all $\phi_{1} \in W^{1,2}\left(t_{0}, \mathcal{T} ; H\right) \cap$ $L^{2}\left(t_{0}, \mathcal{T} ; V\right) \cap L^{4}(Q)$ and all $\phi_{2} \in W^{1,2}\left(t_{0}, \mathcal{T} ; H\right)$

$$
\begin{aligned}
\int_{t_{0}}^{t}\left\{\left(u, \partial_{t} \phi_{1}\right)-a\left(u, \phi_{1}\right)-\left(f(u, w), \phi_{1}\right)\right\} \mathrm{d} \tau & =-\int_{t_{0}}^{t}\left(s(\tau), \phi_{1}(\tau)\right) \mathrm{d} \tau+\left(u(t), \phi_{1}(t)\right)-\left(v\left(t_{0}\right), \phi_{1}(0)\right), \\
\int_{t_{0}}^{t}\left\{\left(w, \partial_{t} \phi_{2}\right)-\left(g(u, w), \phi_{2}\right)\right\} \mathrm{d} \tau & =\left(w(t), \phi_{2}(t)\right)-\left(z\left(t_{0}\right), \phi_{2}\left(t_{0}\right)\right),
\end{aligned}
$$

for all $t \in\left(t_{0}, \mathcal{T}\right)$, and $(u, w)$ satisfies the following strong energy identity:

$$
\left(\|u(t)\|_{H}^{2}+\|w(t)\|_{H}^{2}\right)+2 \int_{t_{0}}^{t} a(u(\tau), u(\tau)) \mathrm{d} \tau+2 \int_{t_{0}}^{t} \int_{\Omega} f(u(\tau), w(\tau)) u(\tau)+g(u(\tau), w(\tau)) w(\tau) \mathrm{d} x \mathrm{~d} \tau
$$

$=\left\|v\left(t_{0}\right)\right\|_{H}^{2}+\left\|z\left(t_{0}\right)\right\|_{H}^{2}+2 \int_{t_{0}}^{t}(s(\tau), u(\tau)) \mathrm{d} \tau$.

Next, denote by

$$
\begin{aligned}
& v_{h}(t):=\int_{0}^{\mathcal{T}} j_{h}(t-\tilde{t}) v(\tilde{t}) \mathrm{d} \tilde{t}, \quad z_{h}(t):=\int_{0}^{\mathcal{T}} j_{h}(t-\tilde{t}) z(\tilde{t}) \mathrm{d} \tilde{t} \\
& u_{h}(t):=\int_{0}^{\mathcal{T}} j_{h}(t-\tilde{t}) u(\tilde{t}) \mathrm{d} \tilde{t}, \quad w_{h}(t):=\int_{0}^{\mathcal{T}} j_{h}(t-\tilde{t}) w(\tilde{t}) \mathrm{d} \tilde{t}
\end{aligned}
$$


the (Friedrichs) time-mollifier of $v, z, u$, and $w$, respectively, where $j_{h} \in \mathrm{C}_{c}^{\infty}(-h, h), 0<h<\mathcal{T}$, is even and positive with $\int_{\mathbb{R}} j_{h}(\tilde{t}) \mathrm{d} \tilde{t}=1$. Then, as is well known,

$$
\begin{aligned}
& \lim _{h \rightarrow 0} \int_{0}^{\mathcal{T}}\left\|v_{h}(\tau)-v(\tau)\right\|_{V}^{2} \mathrm{~d} \tau=0, \quad \operatorname{ess}_{\sup _{t \in[0, \mathcal{T}]}\left\|v_{h}(t)\right\|_{2} \leq \operatorname{ess}_{\sup } \sup _{t \in[0, \mathcal{T}]}\|v(t)\|_{2},} \\
& \lim _{h \rightarrow 0} \int_{0}^{\mathcal{T}}\left\|u_{h}(\tau)-u(\tau)\right\|_{H^{2}}^{2} \mathrm{~d} \tau=0, \quad \operatorname{ess}_{\sup _{t \in[0, \mathcal{T}]}\left\|u_{h}(t)\right\|_{V} \leq \operatorname{ess} \sup _{t \in[0, \mathcal{T}]}\|u(t)\|_{V},}^{\mathcal{T}}\left\|\lim _{h \rightarrow 0} \int_{0}^{\mathcal{T}}\right\| w_{h}(\tau)-w(\tau) \|_{H}^{2} \mathrm{~d} \tau=0 .
\end{aligned}
$$

The weak continuity of $v$ and $u$ implies

$$
\begin{aligned}
& \lim _{h \rightarrow 0}\left(u(t), v_{h}(t)\right)=\lim _{h \rightarrow 0}\left(u_{h}(t), v(t)\right)=(u(t), v(t)), t \geq t_{0}, \\
& \lim _{h \rightarrow 0}\left(w(t), z_{h}(t)\right)=\lim _{h \rightarrow 0}\left(w_{h}(t), z(t)\right)=(w(t), z(t)), t \geq t_{0} .
\end{aligned}
$$

Furthermore since

$$
\begin{aligned}
& \int_{t_{0}}^{t}\left(v, \partial_{t} u_{h}\right) \mathrm{d} \tau=-\int_{t_{0}}^{t}\left(u_{h}, \partial_{t} v\right) \mathrm{d} \tau+\left(u_{h}(t), v(t)\right)-\left(u_{h}\left(t_{0}\right), v\left(t_{0}\right)\right) \\
& \int_{t_{0}}^{t}\left(z, \partial_{t} w_{h}\right) \mathrm{d} \tau=-\int_{t_{0}}^{t}\left(w_{h}, \partial_{t} z\right) \mathrm{d} \tau+\left(w_{h}(t), z(t)\right)-\left(w_{h}\left(t_{0}\right), z\left(t_{0}\right)\right)
\end{aligned}
$$

by taking the limit,

$$
\begin{aligned}
& \lim _{h \rightarrow 0}\left\{\int_{t_{0}}^{t}\left(v, \partial_{t} u_{h}\right)+\left(u_{h}, \partial_{t} v\right) \mathrm{d} \tau\right\}=(u(t), v(t))-\left\|v\left(t_{0}\right)\right\|_{H}^{2} \\
& \lim _{h \rightarrow 0}\left\{\int_{t_{0}}^{t}\left(z, \partial_{t} w_{h}\right)+\left(w_{h}, \partial_{t} z\right) \mathrm{d} \tau\right\}=(w(t), z(t))-\left\|z\left(t_{0}\right)\right\|_{H}^{2} .
\end{aligned}
$$

We now replace $\varphi_{1}$ by $u_{h}$ in (4.2), $\varphi_{2}$ by $w_{h}$ in (4.3), $\phi_{1}$ by $v_{h}$ in (4.5), and $\phi_{2}$ by $z_{h}$ in (4.6). Then, we sum up the resulting equations to obtain

$$
\begin{aligned}
& \int_{t_{0}}^{t}\{-2 a(u, v)-(f(v, z), u)-(f(u, w), v)-(g(v, z), w)-(g(u, w), z)\} \mathrm{d} \tau \\
= & -\int_{t_{0}}^{t}(s(\tau), u(\tau)+v(\tau)) \mathrm{d} \tau+(u(t), v(t))-\left\|v\left(t_{0}\right)\right\|_{H}^{2}+(w(t), z(t))-\left\|z\left(t_{0}\right)\right\|_{H}^{2} .
\end{aligned}
$$

To prove $(u, w)=(v, z)$, we calculate

$$
\begin{aligned}
& \|u(t)-v(t)\|_{H}^{2}+\|w(t)-z(t)\|_{H}^{2}+2 \int_{t_{0}}^{t} a(u(\tau)-v(\tau), u(\tau)-v(\tau)) \mathrm{d} \tau \\
= & \left(\|u(t)\|_{H}^{2}+\|w(t)\|_{H}^{2}+2 \int_{t_{0}}^{t} a(u(\tau), u(\tau)) \mathrm{d} \tau\right)+\left(\|v(t)\|_{H}^{2}+\|z(t)\|_{H}^{2}+2 \int_{t_{0}}^{t} a(v(\tau), v(\tau)) \mathrm{d} \tau\right) \\
& \quad-2(u(t), v(t))-2(w(t), z(t))-4 \int_{t_{0}}^{t} a(u(\tau), v(\tau)) \mathrm{d} \tau .
\end{aligned}
$$

For the first two parts, we use the strong energy equality (4.7) and the strong energy inequality (4.4), and for the last term, we use the relation (4.13). Then, we have

$$
\begin{gathered}
\|u(t)-v(t)\|_{H}^{2}+\|w(t)-z(t)\|_{H}^{2}+2 \int_{t_{0}}^{t} a(u(\tau)-v(\tau), u(\tau)-v(\tau)) \mathrm{d} \tau \\
\leq 2 \int_{t_{0}}^{t}\{(f(v, z), u)+(f(u, w), v)-(f(u, w), u)-(f(v, z), v) \\
\quad+(g(v, z), w)+(g(u, w), z)-(g(u, w), w)-(g(v, z), z)\} \mathrm{d} \tau
\end{gathered}
$$




$$
\leq-2 \int_{t_{0}}^{t}(f(u, w)-f(v, z), u-v)+(g(u, w)-g(v, z), w-z) \mathrm{d} \tau
$$

Here, for the first term we use the Assumption (4.1) and Young's inequality to get

$$
\begin{aligned}
-2 \int_{t_{0}}^{t}(f(u, w)-f(v, z), u-v) \mathrm{d} \tau & \leq 2 \lambda_{f} \int_{t_{0}}^{t}\|u(\tau)-v(\tau)\|_{H}^{2} \mathrm{~d} \tau-2 \int_{t_{0}}^{t}(w-z, u-v) \mathrm{d} \tau \\
& \leq 2 \lambda_{f} \int_{t_{0}}^{t}\|u(\tau)-v(\tau)\|_{H}^{2} \mathrm{~d} \tau+\int_{t_{0}}^{t} \varepsilon_{1}\|w-z\|_{H}^{2}+C\left(\varepsilon_{1}\right)\|u-v\|_{H}^{2} \mathrm{~d} \tau
\end{aligned}
$$

for some constants $\varepsilon_{1}, C\left(\varepsilon_{1}\right)>0$. On the other hand since the function $g(u, w)=-\varepsilon(k u-w)$ is linear,

$$
|(g(u, w)-g(v, z), w-z)| \leq C\left(\|u-v\|_{H}^{2}+\|w-z\|_{H}^{2}\right) .
$$

for some $C>0$. Therefore, we have

$$
\begin{aligned}
& \|u(t)-v(t)\|_{H}^{2}+\|w(t)-z(t)\|_{H}^{2}+2 \int_{t_{0}}^{t} a(u(\tau)-v(\tau), u(\tau)-v(\tau)) \mathrm{d} \tau \\
\leq & C \int_{t_{0}}^{t}\left(\|u(\tau)-v(\tau)\|_{H}^{2}+\|w(\tau)-z(\tau)\|_{H}^{2} \mathrm{~d} \tau,\right.
\end{aligned}
$$

for some $C>0$, which is different from the previous constant.

Hence, we are able to apply Gronwall's lemma to conclude that

$$
u-v \equiv 0, w-z \equiv 0 \text { a.e. in } \Omega \times\left[t_{0}, \mathcal{T}\right] .
$$

This implies the existence of a strong $T$-time-periodic solution $(u, w)$ when the source term $s_{i, e}$ is a $T$-time-periodic function.

We write down the main theorem of the existence of strong periodic solutions without assuming smallness conditions for the external forces.

Theorem 4.5. Let $d=3, T>0$, and $s_{i, e} \in L^{2}(\mathbb{T} ; H)$ with $s_{i}+s_{e} \in W^{1,1}(\mathbb{T} ; H)$ and $\int_{\Omega}\left(s_{i}+s_{e}\right) \mathrm{d} x=0$ for a.e. $t$. Let the conductivity matrices $\sigma_{i, e}$ satisfy the Assumption $\square$ and the nonlinear term $f$ satisfy the Assumption (4.1) and assume that there exist constants $C_{0} \in \mathbb{R}$ and $C_{1}>1$ such that

$$
C_{0}+C_{1}|u|^{4} \leq f(u) u
$$

for all $u \in \mathbb{R}$. Then for the bidomain equations with FitzHugh-Nagumo type

$$
\left\{\begin{aligned}
\partial_{t} u-\operatorname{div}\left(\sigma_{i} \nabla u_{i}\right)+f(u)+w & =s_{i}, & & \text { in }(0, \infty) \times \Omega, \\
\partial_{t} u+\operatorname{div}\left(\sigma_{e} \nabla u_{e}\right)+f(u)+w & =-s_{e}, & & \text { in }(0, \infty) \times \Omega, \\
u & =u_{i}-u_{e}, & & \text { in }(0, \infty) \times \Omega, \\
\partial_{t} w-\varepsilon(k u-w) & =0, & & \text { in }(0, \infty) \times \Omega, \\
\sigma_{i} \nabla u_{i} \cdot \nu=0, \sigma_{e} \nabla u_{e} \cdot \nu & =0, & & \text { in }(0, \infty) \times \partial \Omega, \\
u(0)=u_{0}, & w(0)=w_{0}, & & \text { in } \Omega,
\end{aligned}\right.
$$

there exists a strong T-periodic solution

$$
\begin{gathered}
\left(u_{i}, u_{e}\right) \in\left(W^{1,2}(\mathbb{T} ; H) \cap L^{2}\left(\mathbb{T} ; H^{2}(\Omega)\right)^{2} \text { with } \int_{\Omega} u_{e} \mathrm{~d} x=0 \text { a.e. } t\right. \\
(u, w) \in\left(W^{1,2}(\mathbb{T} ; H) \cap L^{2}\left(\mathbb{T} ; H^{2}(\Omega)\right) \cap L^{4}(\mathbb{T} \times \Omega)\right) \times C^{1}(\mathbb{T} ; H) .
\end{gathered}
$$

Remark 4.6. The Assumption $\mathrm{N}$ of the existence of the weak periodic solutions is replaced by (4.14).

Remark 4.7. We do not treat the ionic models by Rogers-McCulloch and Aliev-Panfilov due to the lack of a suitable global well-posedness result for the initial value problem in the $L^{2}$ setting. 


\section{Appendix}

In this appendix, we check that the three models introduced in Section 1 satisfy the Assumption $\mathbb{N}$, Since the growth conditions (3.2)-(3.4) are trivial as $p=4$, we confirm the condition (3.1).

5.1. FitzHugh-Nagumo model. The FitzHugh-Nagumo type is

$$
\begin{aligned}
& f(u, w)=u(u-a)(u-1)+w \\
& g(u, w)=-\varepsilon(k u-w)
\end{aligned}
$$

with $0<a<1$ and $k, \varepsilon>0$. Then, we are able to calculate as follows $(r=1)$ :

$$
f(u, w) u+g(u, w) w=u^{4}-(a+1) u^{3}+a u^{2}+u w-\varepsilon k u w+\varepsilon w^{2}
$$

and by

$$
\begin{aligned}
\left|(a+1) u^{3}\right| & \leq \frac{1}{8} u^{4}+c_{11}, \\
\left|a u^{2}\right| & \leq \frac{1}{8} u^{4}+c_{12}, \\
|u w| & \leq \frac{1}{8} u^{4}+\frac{\varepsilon}{4} w^{2}+c_{13}, \\
|\varepsilon u w| & \leq \frac{1}{8} u^{4}+\frac{\varepsilon}{4} w^{2}+c_{14},
\end{aligned}
$$

for some $c_{1 i}>0(i=1, \ldots, 4)$, we have

$$
f(u, w) u+g(u, w) w \geq \frac{1}{2} u^{4}+\frac{\varepsilon}{2} w^{2}+c_{1}
$$

for some $c_{1} \in \mathbb{R}$. Therefore, the FitzHugh-Nagumo model satisfies the Assumption $\mathbb{N}$.

5.2. Rogers-McCulloch model. The Rogers-McCulloch type is

$$
\begin{aligned}
& f(u, w)=b u(u-a)(u-1)+u w \\
& g(u, w)=-\varepsilon(k u-w)
\end{aligned}
$$

with $0<a<1$ and $b, k, \varepsilon>0$. Then, we are able to calculate as follows:

$$
r f(u, w) u+g(u, w) w=r b u^{4}-r b(a+1) u^{3}+r b a u^{2}+r u^{2} w-\varepsilon k u w+\varepsilon w^{2}
$$

and, based on the calculation

$$
\left|r u^{2} w\right| \leq \frac{C^{2}}{2} u^{4}+\frac{r^{2}}{2 C^{2}} w^{2},
$$

we choose $r, C>0$ depending on $b, \varepsilon$, such that

$$
\left\{\begin{array}{l}
c_{21}:=r b-\frac{C^{2}}{2}>0, \\
c_{22}:=\varepsilon-\frac{r^{2}}{2 C^{2}}>0 .
\end{array}\right.
$$

By

$$
\begin{aligned}
\left|r b(a+1) u^{3}\right| & \leq \frac{c_{21}}{6} u^{4}+c_{23}, \\
\left|r b a u^{2}\right| & \leq \frac{c_{21}}{6} u^{4}+c_{24}, \\
|\varepsilon k u w| & \leq \frac{c_{21}}{6} u^{4}+\frac{c_{22}}{2} w^{2}+c_{25},
\end{aligned}
$$

for some $c_{2 i}>0(i=3, \ldots, 5)$, we have

$$
r f(u, w) u+g(u, w) w \geq \frac{c_{21}}{2} u^{4}+\frac{c_{22}}{2} w^{2}+c_{2}
$$

for some $c_{2} \in \mathbb{R}$. Therefore, the Rogers-McCulloch model satisfies the Assumption $\mathbb{N}$ 
5.3. Aliev-Panfilov model. The modified Aliev-Panfilov type is

$$
\begin{aligned}
& f(u, w)=b u(u-a)(u-1)+u w \\
& g(u, w)=\varepsilon(k u(u-1-d)+w)
\end{aligned}
$$

with $0<a, d<1, b, k, \varepsilon>0$, and $b>k$. Then, we are able to calculate as follows:

$$
r f(u, w) u+g(u, w) w=r b u^{4}+r b(a+1) u^{3}+r b a u^{2}+r u^{2} w+\varepsilon k u^{2} w-\varepsilon k(1+d) u w+\varepsilon w^{2}
$$

and, based on the calculation

$$
\left|(r+\varepsilon k) u^{2} w\right| \leq \frac{C^{2}}{2} u^{4}+\frac{(r+\varepsilon k)^{2}}{2 C^{2}} w^{2},
$$

we choose $r, C>0$ depending on $b, k, \varepsilon$, such that

$$
\left\{\begin{array}{l}
c_{31}:=r b-\frac{C^{2}}{2}>0, \\
c_{32}:=\varepsilon-\frac{(r+\varepsilon k)^{2}}{2 C^{2}}>0 .
\end{array}\right.
$$

Here, the assumption $b>k$ is essential. By

$$
\begin{aligned}
\left|r b(a+1) u^{3}\right| & \leq \frac{c_{31}}{6} u^{4}+c_{33}, \\
\left|r b a u^{2}\right| & \leq \frac{c_{31}}{6} u^{4}+c_{34}, \\
|\varepsilon k(1+d) u w| & \leq \frac{c_{31}}{6} u^{4}+\frac{c_{32}}{2} w^{2}+c_{35},
\end{aligned}
$$

for some $c_{3 i}>0(i=3, \ldots, 5)$, we have

$$
r f(u, w) u+g(u, w) w \geq \frac{c_{31}}{2} u^{4}+\frac{c_{32}}{2} w^{2}+c_{3}
$$

for some $c_{3} \in \mathbb{R}$. Note that we are not able to take $c_{3 i}(i=1,2)$ in the case $b=k$. Therefore, the modified Aliev-Panfilov model satisfies the Assumption [N]

\section{REFERENCES}

[1] R. Aliev and A. Panfilov, A simple two-variable model of cardiac excitation, Chaos, Solitions \& Fractals. 7 (1996), no. 3, 293-301.

[2] Z. Belhachmi and R. Chill, The bidomain problem as a gradient system, 2018. arXiv: 1804.08272.

[3] Y. Bourgault, Y. Coudière, and C. Pierre, Existence and uniqueness of the solution for the bidomain model used in cardiac electrophysiology., Nonlinear Anal. Real World Appl. 10 (2009), no. 1, 458-482.

[4] P. Colli Franzone, L. Guerri, and S. Tentoni, Mathematical modeling of the excitation process in myocardial tissue: influence of fiber rotation on wavefront propagation and potential field., Math. Biosci. 101 (1990), no. 2, 155-235.

[5] P. Colli Franzone, L. F. Pavarino, and S. Scacchi, Mathematical cardiac electrophysiology., MS\&A. Modeling, Simulation and Applications, vol. 13, Springer, Cham, 2014.

[6] P. Colli Franzone, M. Pennacchio, and G. Savaré, Multiscale modeling for the bioelectric activity of the heart., SIAM J. Math. Anal. 37 (2005), no. 4, 1333-1370.

[7] P. Colli Franzone and G. Savaré, Degenerate evolution systems modeling the cardiac electric field at micro- and macroscopic level., Evolution equations, semigroups and functional analysis (Milano, 2000), 2002, pp. 49-78.

[8] R. FitzHugh, Impulses and Physiological States in Theoretical Models of Nerve Membrane., Biophys. J. 1 (1961), no. $6,445-466$.

[9] M. Hieber, N. Kajiwara, K. Kress, and P. Tolksdorf, Strong Time Periodic Solutions to the Bidomain Equations with FitzFugh-Nagumo Type Nonlinearities., arXiv:1708.05304 (2017).

[10] G. P. Galdi, An introduction to the mathematical theory of the Navier-Stokes equations, Second, Springer Monographs in Mathematics, Springer, New York, 2011. Steady-state problems.

[11] Y. Giga and N. Kajiwara, On a resolvent estimate for bidomain operators and its applications., J. Math. Anal. Appl. 459 (2018), no. 1, 528-555.

[12] G. P. Galdi, M. Hieber, and T. Kashiwabara, Strong time-periodic solutions to the $3 D$ primitive equations subject to arbitrary large forces, Nonlinearity 30 (2017), no. 10, 3979-3992.

[13] M. .O. Gani and T. Ogawa, Stability of periodic traveling waves in the Aliev-Panfilov reaction-diffusion system., Commun. Nonlinear Sci. Numer. Simul. 33 (2016), 30-42.

[14] M. Hieber and J. Prüss, On the bidomain problem with FitzHugh-Nagumo transport., 2018. preprint.

[15] — , L $L_{q}$-theory for the bidomain operator., 2018. preprint. 
[16] N. Kajiwara, Global strong solutions for the bidomain equations with maximal $L_{p, \mu}$ regularity., 2018. preprint.

[17] J. Keener and J. Sneyd, Mathematical physiology., Interdisciplinary Applied Mathematics, vol. 8, Springer-Verlag, New York, 1998.

[18] K. Kunisch and M. Wagner, Optimal control of the bidomain system (II): uniqueness and regularity theorems for weak solutions., Ann. Mat. Pura Appl. (4) 192 (2013), no. 6, 951-986.

[19] Y. Mori and H. Matano, Stability of front solutions of the bidomain equation., Comm. Pure Appl. Math. 69 (2016), no. $12,2364-2426$.

[20] J. M. Rogers and A. D. McCulloch, A collocation-Galerkin finite element model of cardiac action potential propagation., IEEE Trans. Biomed. Eng. 41 (1994), no. 8, 743-757.

[21] L. Tung, A bidomain model for describing ischemic myocardial d-c potentials., PhD Thesis, MIT. (1978).

[22] M. Veneroni, Reaction-diffusion systems for the macroscopic bidomain model of the cardiac electric field., Nonlinear Anal. Real World Appl. 10 (2009), no. 2, 849-868.

Graduate School of Mathematical Sciences, The University of Tokyo, 3-8-1 Komaba, Meguro, Tokyo, 153-8914, JAPAN

Graduate School of Mathematical Sciences, The University of Tokyo, 3-8-1 Komaba, Meguro, Tokyo, 153-8914, JAPAN

Fachbereich Mathematik, Technische Universität Darmstadt, Schlossgartenstr. 7, 64289 Darmstadt, GerMANY

E-mail address: labgiga@ms.u-tokyo.ac.jp

E-mail address: kajiwara@ms.u-tokyo.ac.jp

E-mail address: kkress@mathematik.tu-darmstadt.de 